\title{
¿Podemos mantenernos actualizados en medicina en el siglo XXI?
}

\author{
Gabriel Rada G 1,2,3, Luz María Letelier $\mathbf{S}^{\mathbf{1}, 2,3}$. \\ How can an internist keep updated \\ in the XXIst century?
}

The amount of information in health care has steadily grown in the past years and this trend will probably continue in the near future. Traditional ways to keep updated are no longer sufficient, particularly in broad areas of knowledge as Internal Medicine or Family Medicine. Therefore, it is necessary to use new approaches. The present article describes five proposals for the clinician that tries to maintain updated but feels overwhelmed by the amount of available information (Rev Méd Chile 2009; 137: 701-8).

(Key words: Education, medical; Evidence-Based Medicine; Internet)

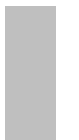

Recibido el 10 de marzo, 2009. Aceptado el 2 de abril, 2009.

${ }^{1}$ Unidad de Medicina Basada en Evidencia, Pontificia Universidad Católica de Chile. Santiago de Chile. ${ }^{2}$ Departamento de Medicina Interna, Pontificia Universidad Católica de Chile. ${ }^{3}$ Unidad Docente Asociada, Hospital Dr. Sótero del Río. Santiago, Chile.

$\mathrm{C}$ omo nunca, esta época se ha caracterizado por el aumento en la cantidad de información disponible y las variadas posibilidades para accederla. El campo de la medicina no es ajeno a esta "sociedad de la información", lo cual se ha manifestado con especial claridad en la última década.

A esta avalancha de información, se agregan las potenciales consecuencias del ejercicio clínico de la medicina sin una actualización permanente. Una revisión sistemática recopiló los estudios que han evaluado el desempeño clínico en relación a los años de egreso. Aproximadamente, $75 \%$ de ellos observaron que el desempeño clínico era peor en todas (50\%) o algunas (25\%) de las áreas evaluadas (desempeño clínico, indicación de terapias sustentadas por evidencia, adherencia a trata-

Correspondencia a: Dr. Gabriel Rada. Módulo Universidad Católica, Hospital Dr. Sótero del Río. Av. Concha y Toro 3459. Fax: 56-2-2897638. E mail: gabriel@rada.cl miento estándar y conocimientos). Si bien se pueden encontrar diversas explicaciones a estos hallazgos, muy probablemente la dificultad en mantenerse al día juega un rol fundamental ${ }^{1}$.

Una forma tradicionalmente utilizada por los clínicos para mantenerse actualizados, es la suscripción a revistas de su especialidad u otras de interés general. Sin embargo, esto como única forma de actualización, parece una opción poco eficiente. A modo de ejemplo, MEDLINE, la principal base de datos de revistas biomédicas, cuenta con más de 18.000.000 artículos, con más de 700.000 nuevas incorporaciones cada año ${ }^{2}$. Obviamente, no todo lo que se publica es relevante al quehacer de cada profesional. En este sentido, uno de los principales aportes de la Medicina Basada en Evidencia (MBE) ha sido el consenso acerca de lo que se ha llamado la jerarquía de la evidencia, en la cual a ciertos diseños de estudio con menor riesgo de sesgo se les asigna un mayor valor. En lo que respecta a las decisiones terapéuticas, los estudios clínicos randomizados (ECR) constituyen mejor evidencia 
que los estudios observacionales y, a su vez, una revisión sistemática (RS) de ECRs se considera de mayor jerarquía que un ECR individual ${ }^{3}$. Aun si consideramos como única fuente de actualización sólo los estudios de mayor jerarquía, la situación sigue siendo inabordable. Siguiendo con el ejemplo de MEDLINE, sólo en esta base de datos se han publicado más de 18.000 ECRs y 2.600 RSs en el último año ${ }^{2}$. A este gran volumen de información se debe agregar la dificultad de muchos clínicos (particularmente aquellos alejados de la investigación) para analizar críticamente la información desde un punto de vista metodológico, y la dificultad de leer lo que se publica en idioma inglés ${ }^{4}$.

Finalmente, otras estrategias frecuentes de actualización, como cursos de formación continua, cursos en línea o asistencia a congresos, también han probado ser poco eficientes ${ }^{5}$.

En el presente artículo se presentan 5 propuestas que conjugan conceptos de educación médica, MBE y el aprovechamiento de las nuevas oportunidades que ofrece Internet y que en opinión de los autores son cardinales para lograr mantenerse actualizado en forma eficiente..." sin morir en el intento".

Propuesta 1: Responda a preguntas clínicas específicas. Es claro que el aprendizaje requiere de una activación previa, es decir, de algún elemento motivador que despierte nuestro interés, de mane- ra que al enfrentarnos al nuevo conocimiento, lo asimilemos mejor. También se sabe que el compromiso emocional con lo aprendido, aumenta la posibilidad de adquirirlo, en el largo plazo. Por último, la aplicación en la práctica es fundamental para integrar los nuevos conocimientos en forma efectiva a nuestro quehacer.

Este ciclo activación-adquisición-aplicación ("las $3 \mathrm{~A}$ ") se cumple a la perfección en la dinámica de la relación médico-paciente. Un paciente motiva una duda clínica (activación), mediante una búsqueda obtenemos la información más relevante (adquisición) y finalmente utilizamos lo aprendido en el mismo paciente 0 en situaciones similares (aplicación).

Algunos sitios en la web ofrecen un servicio que replica en cierta medida este proceso, mediante preguntas en relación a un caso clínico. $\mathrm{Si}$ bien constituyen una excelente herramienta, debe destacarse que el ciclo de las " $3 \mathrm{~A}$ " se realiza en un orden levemente distinto (activación-aplicación-adquisición) lo que lo hace menos eficiente que la situación clínica real (Tabla 1).

La gran mayoría de las preguntas que nos hacemos los médicos, tienen relación con la toma de decisiones clínicas (terapéuticas y diagnósticas, principalmente). La importancia cardinal de formularse preguntas adecuadas, es reconocida por prácticamente todas las disciplinas del saber. La Medicina Basada en Evidencia (MBE) no es la excepción, pues propone iniciar el proceso de

Tabla 1. Sitios web basados en casos clínicos o pacientes simulados

\begin{tabular}{|ll|}
\hline Recurso & Comentarios \\
\hline MD Choice: & Simulador de pacientes. Un interesante formato \\
http://www.mdchoice.com/cyberpt/cyber.asp & interactivo para casos clínicos. \\
NEJM Image Challenge: & Tradicional serie de imágenes del New \\
http://image-challenge.nejm.org/ & England Journal of Medicine. \\
& Está orientado a médicos con experiencia, por lo que \\
& se encuentra enfocado en patologías poco frecuentes y \\
& no da información posterior. \\
eMedicine CME Case Presentations: & Excelente sitio, con casos variados, preguntas de \\
http://cme.medscape.com/index/list_3286_0 0 & múltiple elección y buena información de apoyo luego \\
& de la respuesta. \\
& Es gratuito pero requiere suscripción. Permite recibir \\
& "el caso de la semana" vía correo electrónico. \\
&
\end{tabular}


resolución de una toma de decisión clínica, transformando la duda en una pregunta clínica estructurada ${ }^{6}$. El siguiente paso es realizar una búsqueda eficiente (que se discute en la propuesta 2), seguido del análisis crítico de la información encontrada y finalmente la decisión de aplicar o no la evidencia, a la situación clínica que motivó el proceso ${ }^{7,8}$. Así, constituye un proceso que se adapta muy bien a las "3 A". Para quienes deseen profundizar en el proceso de la MBE, el sitio web de nuestra Unidad de Medicina Basada en Evidencia cuenta con un tutorial en línea que explica este y otros aspectos de la toma de decisiones clínicas basadas en evidencia (http://www.umbeuc.cl/manualonline/index.html).

Propuesta 2: Realice una búsqueda eficiente de la literatura. Debido a la proliferación de información, tanto en revistas médicas como en otras fuentes, cada vez más se requiere de habilidades específicas para buscar eficientemente la información relevante.

Desde el punto de vista de la relación del clínico con la información, uno de los principales aportes de la MBE es la generación de evidencia filtrada, en donde la investigación original es analizada críticamente y entregada en forma de resumen estructurado. Algunos ejemplos de esta evidencia filtrada son las síntesis y las sinopsis de la evidencia (Tabla 2).

La forma más completa de síntesis la constituyen las revisiones sistemáticas (revisión de una pregunta clínica específica utilizando métodos explícitos para identificar, seleccionar y evaluar críticamente los estudios relevantes. Puede utilizar o no métodos estadísticos, como metaanálisis). Una buena RS nos permite tener acceso a la mayor parte de la información disponible (publicada y no publicada) sobre una pregunta clínica, lo cual es prácticamente imposible de realizar por un solo individuo enfrentado a múltiples incertidumbres durante su quehacer clínico. En la actualidad, la principal fuente de RSs es la Colaboración Cochrane. Sus productos se caracterizan por la transparencia del proceso, un alto nivel de calidad y el compromiso de sus autores de mantener actualizadas las revisiones ${ }^{9}$. El Centro Cochrane Iberoamericano traduce la versión original de todas las RSs Cochrane, en una iniciativa llamada Cochrane Library Plus, a la que se puede acceder en forma gratuita a través del sitio de la biblioteca virtual en salud (http:// www.bireme.br).

Las sinopsis son un poco más simples, pero pueden generarse más rápidamente. Corresponden a artículos o series de artículos analizados críticamente y resumidos en un formato estructurado. Permiten ahorrar tiempo, en comparación con una búsqueda y análisis crítico de la investigación original. Muchas revistas médicas publican periódicamente este tipo de recursos (por ejemplo la sección "Análisis crítico de un artículo" de la Revista Médica de Chile). Adicionalmente, existen revistas dedicadas sólo a la publicación de sinopsis, denominadas revistas secundarias (por ejemplo el ACP Journal Club del American College of Physicians). Existen algunos sitios web que producen un mayor número de sinopsis, aunque sin un proceso editorial tan estandarizado como las revistas. El año 2001, en nuestra Unidad, adaptamos los formatos internacionales de sinopsis de la evidencia, creando el formato ECLIPSE (Enfoque Clínico del Paciente Sustentado por Evidencia) que constituye una herramienta muy útil para la docencia y difusión de la $\mathrm{MBE}^{10}$.

Actualmente existen múltiples sitios donde buscar síntesis y sinopsis de la evidencia. Para poder acceder a éstos y otros recursos de interés, se han creado recursos denominados Metabuscadores, que permiten desde un único lugar realizar búsquedas en múltiples recursos relevantes desde el punto de vista de la MBE (Figura 1). Este tipo de recurso se ha transformado en el mejor lugar para iniciar una búsqueda que responda una pregunta clínica específica y sólo debiéramos utilizar otros sitios cuando no encontremos respuestas con un metabuscador (Tabla 3).

A pesar de la existencia de recursos que nos permiten encontrar rápidamente información filtrada, en muchas ocasiones tendremos que recurrir a la búsqueda en una base de datos, especialmente cuando queramos responder a preguntas sobre las cuales existe poca información. Desde el punto de vista del clínico, hay pocas razones para utilizar una base de datos diferente a PubMed/MEDLINE (MEDLINE es el nombre de la base de datos y PubMed la interfase, es decir el sitio desde donde la accedemos. Si bien se puede buscar en MEDLINE desde otras interfases, PubMed es actualmente la más completa y es gratuita). Como consecuencia de la gran cantidad de 
Tabla 2. M etabuscadores, síntesis y sinopsis de la evidencia

\begin{tabular}{|ll|}
\hline Recurso & Comentarios \\
\hline $\begin{array}{l}\text { Metabuscadores } \\
\text { TRIP database: }\end{array}$ & Permite la búsqueda simultánea en más de 90 sitios relevantes \\
$\begin{array}{l}\text { http://www.tripdatabase.com database (versión en español) } \\
\text { http://excelenciaclinica.net/ }\end{array}$ & $\begin{array}{l}\text { para la MBE. La versión en español no sólo permite buscar } \\
\text { sino también acceder a síntesis, sinopsis y otros recursos }\end{array}$ \\
SUMSearch: & Realiza búsqueda con múltiples opciones según el tipo de \\
http://sumsearch.uthscsa.edu/ & evidencia que se requiera y adapta la búsqueda automática- \\
& mente dependiendo del número de páginas encontradas, \\
& para entregar una cantidad acotada.
\end{tabular}

Sinopsis de evidencia

ACP Journal Club:

http://www.acpjc.org/

EBM online:

ebm.bmj.com/

EBM online (versión en español)

http://ebm.isciii.es/

Revistas secundarias, publicadas colaborativamente por el American College of Physicians, American College of Internal Medicine y el BMJ Group.

Presentan sinopsis de artículos de Medicina Interna general y sus subespecialidades, seleccionados de más de 100 revistas.

Bandolier:

Publicación online, producida por académicos de la Universidad http://www.medicine.ox.ac.uk/bandolier/ Bandolier (versión en español): de Oxford. Escrito en forma amena, entrega una sinopsis de http://www.infodoctor.org/bandolera/ la evidencia en torno a una pregunta, incorporando muchas veces más que un único artículo.

Síntesis de evidencia

La Colaboración Cochrane:

http://www.cochrane.org

Sitio oficial de la colaboración, con información acerca de su http://www.thecochranelibrary.com

http://cochrane.bvsalud.org/portal funcionamiento y muchos otros recursos.

Sitio donde se publican las revisiones sistemáticas y otros productos de la Colaboración Cochrane.

La Cochrane Library Plus es la versión en español de la Cochrane Library. Gratuita.

Clinical Evidence:

http://www.clinicalevidence.com

Libro de resúmenes de evidencia.

También traducido al español.

\section{Tabla 3. ¿Por qué iniciar la búsqueda en un metabuscador?}

- Nos permiten acceder a síntesis y sinopsis de la evidencia, las cuales no siempre están disponibles ni siquiera en las bases de datos como MEDLNE.

- Son más fáciles de utilizar y entregan respuestas más rápidas.

- La información que entregan es preferentemente información filtrada.

- Entregan información jerarquizada, dando preferencia a aquella más relevante desde el punto de vista de la MBE.

- Los principales metabuscadores son gratuitos. 


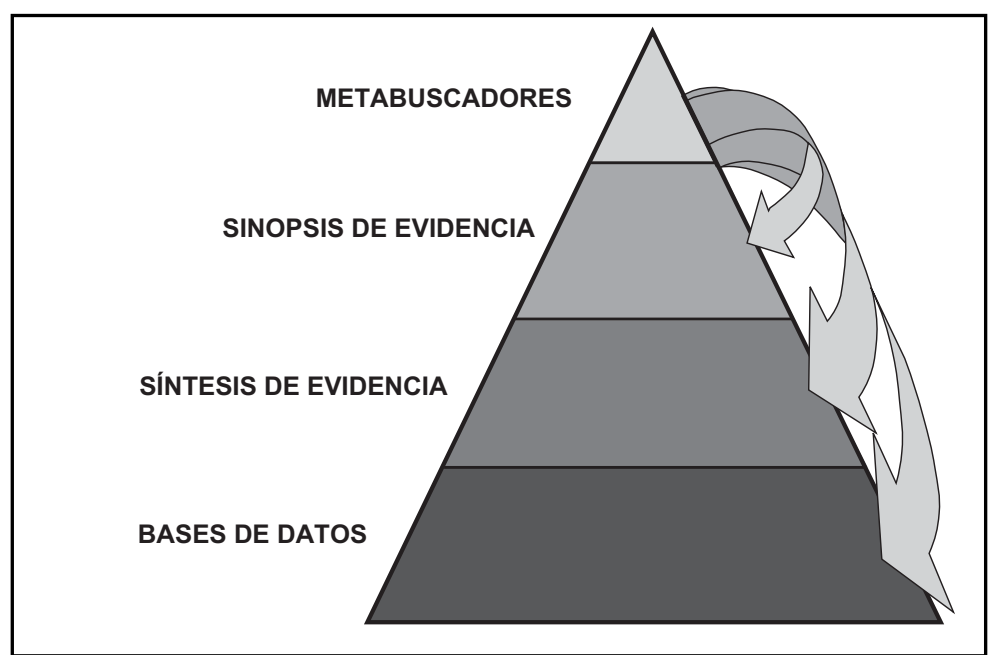

Figura 1. Organización de los recursos de búsqueda: Los metabuscadores permiten acceder a las síntesis y sinopsis de la evidencia, así como también a las bases de datos, pero utilizando filtros. El ancho de la pirámide refleja la cantidad de información que tienen estos recursos.

información contenida en esta base de datos, realizar una búsqueda, por muy simple que sea, puede ser muy poco eficiente si no se tienen las herramientas necesarias.

Realizar un breve entrenamiento en la utilización de PubMed puede significar un enorme ahorro de tiempo a futuro. Si bien los cursos presenciales pueden ser más completos y efectivos (existe una variada oferta de cursos), hay también buenas alternativas de entrenamiento en línea (Tabla 4).

Propuesta 3: Suscríbase a sistemas de ALERTA. Revisar las revistas de la especialidad de interés es una de las formas más tradicionales de mantenerse al día. Dado que la lectura de la revista completa es difícil, algunos intentan hojear la tabla de contenidos y seleccionar algunos artículos. Si esto ya era difícil de lograr hace algunos años, con el aumento en la cantidad de información, en la actualidad se ha vuelto prácticamente imposible revisar todo lo relevante. A modo de ejemplo, para la elaboración de la revista secundaria ACP Journal Club, de 60.000 artículos revisados en un año (provenientes de la revisión manual de 170 revistas relacionadas con Medicina Interna), 3.000 fueron considerados relevantes ${ }^{11}$. En pocas palabras, si quisiéramos leer todo lo relevante publicado, tendríamos que revisar más de 8 artículos al día.

Para solucionar este problema se han creado los llamados sistemas de ALERTA. Un grupo de personas revisa las distintas revistas, seleccionando aquellos artículos de mayor interés, los cuales

Tabla 4. Recursos de entrenamiento en línea para el uso de PubMed/M ED LIN E (http:// www.pubmed.com)

Tutorial oficial de PubMed:

http://www.nlm.nih.gov/bsd/ disted/pubmedtutorial/

Tutorial en español:

http://nnlm.gov/training/resources/intlpubmedlinks.html 
son subidos a una página web o distribuidos por correo electrónico.

Las características más importantes para seleccionar un sistema de ALERTA (empiece con uno o dos como máximo) son la cobertura, es decir, la lista de revistas que son revisadas y los criterios empleados para la selección de artículos, que generalmente dependen de la calidad metodológica o relevancia clínica. También puede considerar al elegir un sistema de alerta aquellos que le permiten personalizar la información que recibirá según su área de interés. Nuestra Unidad produce el "ALERTA UMBE" que desde hace 5 años se distribuye en forma gratuita, mensualmente $\mathrm{y}$ cubre más de 65 revistas, constituyéndose en el mayor alerta disponible en español. En la Tabla 5 se describen las características de los principales sistemas de alerta.

Propuesta 4: Personalice y automatice la información que recibe. Una alternativa a la distribución de información por correo electrónico son los sistemas de alimentación automática (por ej. el formato Really Simple Syndication o RSS). Desde un mismo sitio o programa usted puede tener acceso rápido a toda la información seleccionada, la cual será enviada automáticamente cada vez que el proveedor de la información la actualice. Estos sistemas están compuestos básicamente por
2 elementos: el sistema personalizado (agregador), y los sitios que lo alimentan.

Existen softwares independientes que funcionan como agregadores, sin embargo, lo más fácil es utilizar aquellos basados en la web. Si usted es dueño de una cuenta de correos en alguno de los grandes proveedores (Google, Yahoo, Hotmail, etc.), lo más simple será adicionar esta funcionalidad a su cuenta. Por ejemplo: Usted puede crear su sistema personalizado en iGoogle y suscribirse a una revista de su especialidad. Cada vez que la tabla de contenidos de la revista se actualice, será enviada a su sistema personalizado.

En la Tabla 6 se encuentran las direcciones de los principales agregadores asociados a proveedores de correo electrónico (empezar a utilizarlos es sumamente sencillo y autoexplicativo). Si bien la oferta de contenidos en salud aún no es tan amplia, podemos encontrar las tablas de contenidos y otros materiales producidos por las principales revistas médicas, además de portales de noticias y avances en salud.

Propuesta 5: Familiarícese con la Web 2.0 y aproveche sus enormes potencialidades. El concepto de Web 2.0 se utiliza para definir la web basada en comunidades de usuarios, es decir, cualquier sitio que permita al usuario agregar nuevo contenido o modificar el ya existente. En general este tipo de recursos han

Tabla 5. Principales sistemas de alerta

\begin{tabular}{|c|c|c|}
\hline Alerta & Cobertura & Comentarios \\
\hline $\begin{array}{l}\text { Evidence Updates } \\
\text { http://bmjupdates.mcmaster.ca }\end{array}$ & Más de 150 revistas & $\begin{array}{l}\text { Alerta producido por el grupo editorial BMJ } \\
\text { y la Universidad de McMaster. Metodoló- } \\
\text { gicamente es el más riguroso y el proce- } \\
\text { so de asignación de relevancia es } \\
\text { efectuado por un grupo multinacional } \\
\text { de profesionales. }\end{array}$ \\
\hline $\begin{array}{l}\text { Journal Watch } \\
\text { http://www.jwatch.org }\end{array}$ & Más de 180 revistas & $\begin{array}{l}\text { Producido por el grupo que publica el New } \\
\text { England Journal of Medicine. No sólo } \\
\text { entrega alertas sino otros servicios rela- } \\
\text { cionados. No todos son gratuitos. }\end{array}$ \\
\hline $\begin{array}{l}\text { ALERTA UMBE } \\
\text { http://www.umbeuc.cl/alertas.php }\end{array}$ & Más de 65 revistas & $\begin{array}{l}\text { Producido por la Unidad de Medicina } \\
\text { Basada en Evidencia de la Pontificia } \\
\text { Universidad Católica de Chile. Es el } \\
\text { alerta más completo en castellano. }\end{array}$ \\
\hline
\end{tabular}


Tabla 6. Contenidos de alimentación automática (RSS)

\begin{tabular}{|l|}
\hline Principales agregadores basados en la web \\
\hline iGoogle: http://www.google.com/ig \\
My Yahoo: http:// es.my.yahoo.com/ \\
Live.com: http://www.live.com/ \\
\hline
\end{tabular}

experimentado un aumento exponencial en los últimos años y ya contamos con sitios especialmente diseñados para profesionales médicos.

Existen varias categorías dentro del concepto de Web 2.0, de las cuales mencionaremos las más importantes y algunos ejemplos relevantes en salud (Tabla 7).

Redes sociales: Son sitios en que los usuarios crean información y la comparten con el resto de los miembros, existiendo la posibilidad de interactuar de múltiples formas. El ejemplo más conocido es Facebook, sin embargo, su utilidad en medicina es todavía limitada. Existe también una red social específicamente creada para académi$\cos$ (academia.edu).

Los wikis: Estos sitios permiten a los usuarios editar el contenido de los distintos temas que se agregan al sitio (siguiendo ciertas reglas). El ejemplo más exitoso es Wikipedia, sin embargo se ha criticado que la forma en que se agregan contenidos es demasiado caótica y que el control editorial es muy laxo como para asegurar la calidad de ellos. Como respuesta a esto, un grupo de prestigiosas escuelas de medicina estadounidenses está creando Medpedia, un sitio que pretende convertirse en el principal recurso de información general en medicina.

Marcadores sociales: En éstos, el usuario puede ir "marcando" páginas web mientras navega, las cuales son visibles por el resto de los usuarios que comparten los mismos intereses. Existen sitios especialmente orientados a compartir publicaciones médicas, los cuales constituyen una de las herramientas más promisorias para mantenerse al día.

\section{ConcLusión}

La información médica aumenta constantemente y se hace inabarcable utilizando el enfoque tradicional de actualización. En la actualidad existen herramientas, fundamentalmente provenientes de los principios de la MBE y del desarrollo de Internet, que nos permiten mejorar nuestra eficiencia en esta tarea. No pretendemos ser excluyentes de otras opciones, sin embargo, consideramos que las propuestas descritas son complementarias entre sí, y en nuestra experiencia constituyen una invaluable ayuda al clínico que quiera mantenerse actualizado para beneficio de sus pacientes.

\section{Tabla 7. Web 2.0 en medicina}

\begin{tabular}{|ll|}
\hline Tipo de recurso & Principales ejemplos \\
\hline Redes sociales & Facebook: \\
& http://www.facebook.com \\
& Academia.edu: \\
& http://academia.edu/ \\
Wikis & Wikipedia: \\
& htpp://www.wikipedia.org/ \\
& Medpedia: \\
& http://www.medpedia.com/ \\
Marcadores sociales & Connotea: \\
& http://www.connotea.org/ \\
& CiteUlike: \\
& http://www.citeulike.org/ \\
\hline
\end{tabular}




\section{REFERENCIAS}

1. Choudhry NK, Fletcher RH, Soumeral SB. Systematic review: the relationship between clinical experience and quality of health care. Ann Intern Med 2005; 142: 260-73.

2. Pubmed. Disponible en: www.ncbi.nlm.nih.gov/ pubmed/ [Consultado el 29 de enero de 2009].

3. Guyatt G, Haynes B, Jaeschke R, Meade MO, Wilson M, Montori V, Richardson S. The Philosophy of Evidence-Based Medicine. En: Guyatt G, Rennie D. Users' Guides to the Medical Literature: A Manual for Evidence-Based Clinical Practice, 2a Edición. Editorial McGraw-Hill 2008; 9-16.

4. Leteler LM, Zamarin N, Andrade M, Gabrielu L, Caiozzi G, Viviani P, Riquelme A. Exploring language barriers to Evidence-based Health Care (EBHC) in post-graduate medical students: a randomised trial. Educ Health (Abingdon) 2007; 20: 82-7.

5. Вцоом BS. Effects of continuing medical education on improving physician clinical care and patient health: a review of systematic reviews. Int J Technol Assess Health Care 2005; 21: 380-5.

6. Sото M, Rada G. Formulación de Preguntas en Medicina Basada en la Evidencia. Rev Méd Chile 2003; 131: 1202-7.

7. LeTeler LM, Moore P. La Medicina Basada en Evidencia. Visión después de una década. Rev Méd Chile 2003; 131: 939-46.

8. Rada G, Andrade M. ¿Debo aplicar los resultados de este estudio a mi paciente? Rev Méd Chile 2006; 134: 115-19.

9. About the Cochrane Collaboration. www.cochrane.org/ docs/descrip.htm [Consultado el 29 de enero de 2009].

10. Sitio web UMBE. Qué es un ECLIPSE. www.umbeuc.cl/eclipse que son.html [Consultado el 29 de enero de 2009].

11. Eady A, Glasziou P, Haynes B. Less is more: where do the abstracts in the EBM journal come from? Evid Based Med 2008; 13: 3. 